Relations industrielles

Industrial Relations

\title{
Normes et déclarations de principes intéressant particulièrement les travailleuses, B.I.T., Genève, 1981, 138 pp.
}

\section{Alain Barré}

Volume 36, numéro 3, 1981

URI : https://id.erudit.org/iderudit/029193ar

DOI : https://doi.org/10.7202/029193ar

Aller au sommaire du numéro

Éditeur(s)

Département des relations industrielles de l'Université Laval

ISSN

0034-379X (imprimé)

1703-8138 (numérique)

Découvrir la revue

Citer ce compte rendu

Barré, A. (1981). Compte rendu de [Normes et déclarations de principes intéressant particulièrement les travailleuses, B.I.T., Genève, 1981, 138 pp.] Relations industrielles / Industrial Relations, 36(3), 696-697.

https://doi.org/10.7202/029193ar

Tous droits réservés (C) Département des relations industrielles de l'Université Laval, 1981
Ce document est protégé par la loi sur le droit d'auteur. L'utilisation des services d'Érudit (y compris la reproduction) est assujettie à sa politique d'utilisation que vous pouvez consulter en ligne.

https://apropos.erudit.org/fr/usagers/politique-dutilisation/ 
between women's labour market and family roles and thereby lessen the effectiveness of equal pay legislation. In contrast, Rita Liljestrom discusses the integration of Swedish family policy and labour market policy based on a conception of the family as a symmetrical unit, with two parents and two breadwinners.

What resides after reading this book is a somewhat pessimistic view as to the ultimate success of equal employment policy in most countries. Apart from selecting approaches which are least controversial and most in line with status - quo interests, many countries' experiences have demonstrated the strength of the economic imperative in determining how fare equal employment policy will advance. Various articles point out different approaches toward working women which appear in a growing economy versus one which is declining.

The "reserve army" concept remains strong, with gains for women in the labour market coming mainly in times of economic expansion. As Ralph Smith points out, it becomes increasingly difficult without a healthy labour market to increase women's share of employment in predominantly male sectors. Given the dismal prognosis in most countries in terms of strong and continued economic expansion, the outlook for successful equal employment policy appears rather bleak.

Ratner's final paragraph underlines the importance of this book:

"The development of equal employment policy for women is at a crucial turning point in most of the advanced industrial democracies. Over the next two decades, the parameters of policy in most countries will not only take form, but will also become more difficult to modify." (p. 438)

Clearly, analysis and understanding of the successes and failures of equal employment policy in other countries provide insights which will hopefully be used to im- prove and expand existing policies. Equal Employment Policy For Women is a valuable first step in this direction.

\section{Carole SWAN}

Economic Research and Analysis

Status of Women Canada

\section{Normes et déclarations de principes intéres- sant particulièrement les travailleuses, B.I.T., Genève, 1981, 138 pp.}

Ce recueil rassemble en un seul document diverses normes et déclarations de principes adoptées sous les auspices de l'Organisation internationale du travail et intéressant particulièrement les travailleuses. Il ne comporte pas cependant tous les textes adoptés en la matière: «lorsqu'une norme ou un principe se retrouve dans plusieurs textes, seul le plus important, le plus complet ou le plus à jour est cité» (à la page 2). Des notes placées à la fin de chacune des sections du recueil renvoient aux autres textes pertinents.

En plus d'une importante section regroupant des textes de portée générale concernant l'élimination de la discrimination et la promotion de l'égalité de chances et de traitement ainsi que des textes de portée plus limitée concernant l'égalité de rémunération entre hommes et femmes, ce recueil contient aussi des sections sur la politique de l'emploi et la mise en valeur des ressources humaines, les conditions de travail, la sécurité sociale, les relations professionnelles et l'administration du travail.

Parmi ces divers textes, il importe d'attirer l'attention du lecteur sur les normes dont l'objet est d'assurer une protection spéciale à la femme - les normes protectrices - notamment dans des matières comme le travail de nuit et les travaux insalubres ou dangereux (aux pages 69 et ss.). Or, les normes protectrices sont aujourd'hui fortement remises en question car elles contribueraient selon certains à perpétuer des discriminations à l'égard des femmes. L'O.I.T. n'est pas demeurée insensible à ce point de vue. Une importante 
Déclaration sur l'égalité de chances et de traitement pour les travailleuses (aux pages 14 à 20 , et 77-78) ainsi qu'une Résolution concernant un plan d'action en vue de promouvoir l'égalité de chances et de traitement pour les travailleuses (aux pages 20 à 28, et 78), toutes deux adoptées par la Conférence internationale du travail en 1975, enregistrent l'évolution des idées à ce sujet. Selon l'article 9 par. 2 de la Déclaration, les femmes devront en principe être protégées contre les risques inhérents à leur emploi et à leur profession sur la même base et selon les mêmes normes de protection que les hommes. Les seules mesures de protection spéciale en faveur des femmes ne devraient être prises, aux termes de l'article 9 par. 4, "que pour les types de travail dont il est prouvé qu'ils peuvent leur être préjudiciables du point de vue de leur fonction sociale de reproduction.» De plus, toujours selon le même paragraphe, "ces mesures seront révisées et mises à jour périodiquement, compte tenu des progrès dans les connaissances scientifiques et techniques." L'article 6 du plan d'action précise même qu'elles pourront, le cas échéant, être abrogées selon les besoins et les circonstances de chaque pays.

C'est donc à la lumière de ces principes qu'il faut aujourd'hui lire les mesures de protection spéciale en faveur des femmes contenues dans ce recueil. Cela s'impose d'autant plus que l'adoption de bon nombre de ces mesures remontent déjà à une époque fort lointaine. Dans un pays comme le Canada, où le travail s'exécute dans des conditions relativement satisfaisantes, les normes protectrices en faveur des femmes peuvent revêtir un caractère discriminatoire en raison de leur manque de justification objective.

Cette publication du B.I.T. ne pouvait intervenir à un moment plus opportun puisqu'elle suit de quelques mois seulement l'adoption d'un important programme d'action par la Conférence mondiale de la Décennie des Nations Unies pour la femme: égalité, développement et paix (doc. A/CONF. 94/35) tenue à Copenhague du 14 au 30 juillet 1980. Le paragraphe 121 de ce programme d'action recommande que des mesures spéciales soient prises pour faire ratifier et appli- quer les conventions et recommandations pertinentes de l'O.I.T. concernant les droits de la femme dans le domaine de l'emploi.

Compte tenu de la mise en garde que nous avons formulée à propos des normes protectrices, ce recueil pourra servir de guide à ceux qui élaborent les politiques nationales en matière sociale. Il pourra aussi servir de référence à tous ceux qui s'intéressent à l'égalité de chances et de traitement en faveur des travailleuses.

Alain BARRÉ

Université Laval

Les moyens organisationnels de prévention des maladies industrielles et des accidents du travail: essai de typologie et bibliographie annotée, par René Boulard et Lyne Pérusse, Québec, Collection instruments de travail, Département des relations industrielles, Université Laval, 1981, $183 \mathrm{pp}$.

Dans sa collection instruments de travail, le Département des relations industrielles de l'Université Laval nous présente une typologie sur les moyens organisationnels de prévention des maladies industrielles et des accidents de travail. À tous ceux qui en santé et sécurité du travail recherchent un moyen d'organiser l'information et à tous ceux qui doivent entreprendre l'étude d'un moyen de prévention, René Boulard et Lyne Pérusse rendent service.

En effet, l'ouvrage de Boulard et Pérusse frappe par la clarté de sa présentation et par l'exhaustivité du recensement des moyens organisationnels de prévention. Nous trouvons une table des matières (11 pages), cinq tableaux illustrant le plan de classification, la définition de la typologie, une bibliographie annotée et enfin, un index des auteurs et organismes; le tout avec les renvois nécessaires et Dieu merci avec les mêmes numéros de têtes de chapitre. Le plan général de classification est à quatre niveaux et va du général au particulier, soit: le niveau gouvernemental, les corps intermédiaires, le niveau de l'entre- 